\title{
Ruta de la gestión para la reducción del riesgo de desastres en Honduras
}

Oscar Elvir*, Nabil Kawas*

\section{RESUMEN}

Este trabajo intenta explicar los inicios y estado actual de la Gestión del Riesgo (GR) en Honduras, con el propósito de tener una visión de su evolución y el fortalecimiento de acciones que contribuyan a la reducción de impactos de los desastres. El método de investigación consistió en el uso de entrevistas a coordinadores de proyectos en GR, visitas y entrevistas con directores de instituciones gubernamentales e internacionales que trabajan en GRRD, consultas a bibliografía disponible en lo referente a la temática y experiencia de los autores en la elaboración de diferentes trabajos en GRRD.

Los resultados obtenidos ayudan a comprender el estado de la GRRD en el país en diferentes áreas de interés como la evolución del marco jurídico de las instituciones que trabajan en el tema, las debilidades y fortalezas, los trabajos de investigación realizados, las amenazas de fenómenos naturales y antropogénicos que han causado mayores daños y pérdidas, el cambio climático, la educación, las zonas más vulnerables ante la ocurrencia de frecuentes amenazas y la preparación del país para hacerle frente a un desastre de grandes proporciones y, para finalizar, se discuten los resultados acerca de las directrices que debería tomar la GRRD en el país.

Palabras clave: Gestión para la reducción del riesgo de desastres (GRRD), amenaza, vulnerabilidad, riesgo, cambio climático, impacto, capacidad de respuesta a desastres.

\section{ABSTRACT}

This work attempts to explain the beginnings and current state of the Disaster Risk Reduction Management in Honduras, with the purpose of having a vision of its development in order to take actions that could help to reduce the impacts of disasters. The qualitative research method used here involved the use of interviews

*Universidad Nacional Autónoma de Honduras. Facultad de Ciencias. Instituto Hondureño de Ciencias de la Tierra. 
to coordinators of disaster risk reduction management projects, and also with directors of governmental and international institutions working in the subject, consultation of available literature, and also experiences of the authors working in this topic.

The results help to understand the current state of the disaster risk reduction in the country in different areas of interest such as the evolution of the legal framework, what are the institutions working in GRRD in Honduras, capacity building, weaknesses, previous research works in the subject, hazards that have caused great damage and losses, climate change, education, to determine the most vulnerable regions to the occurrence of frequent hazards and the preparedness of the country to cope with a disaster of major proportions. Finally, we discuss the guidelines that should be taken in the GRRD in the country.

Key words: Risk Management (RG), hazard, vulnerability, risk, climate change, impact, disaster response capability. 


\section{INTRODUCCIÓN}

La Gestión del Riesgo es base esencial en países donde la frecuente ocurrencia de eventos impacta los diferentes sectores de la economía; tal es el caso de Honduras, que año con año se ve afectado por pequeños y medianos desastres provocados por diferentes amenazas. La gestión de riesgos de desastres es un conjunto de medidas de gestión (acciones políticas, técnicas, legales y administrativas orientadas a lograr un mejor manejo de recursos, territorios y actividades dentro de un ámbito geográfico-administrativo determinado) destinadas a reducir los riesgos de desastres (COSUDE, 2002).

Se estima que solo en el año 2,010 las pérdidas por desastres en diferentes sectores de la economía del país fueron de aproximadamente de 68 millones de dólares americanos en el municipio del Distrito Central y en los departamentos de Choluteca y Valle (Motiño, et al. 2010), lo que indica que falta mucho por hacer en el país en materia de GR. En este contexto, el objetivo de este trabajo es dar a conocer cómo han evolucionado las bases para el establecimiento de la GR en el país y su contribución en la reducción de los impactos de los desastres, ofreciendo una estrategia de directrices y acciones necesarias a implementar a corto y mediano plazo.

\section{LOS MÉTODOS}

Este trabajo es del tipo cualitativo, basado en entrevistas y visitas realizadas a Instituciones Nacionales -en especial a la Comisión Permanente de Contingencias (COPECO)—, a instituciones miembros del Sistema Nacional de Gestión del Riesgo de Honduras (SINAGER), a personal de las Organizaciones No Gubernamentales (ONG's) y Universidades.

Se puso especial énfasis en la revisión de documentos relacionados con la temática, especialmente los elaborados para los planes de acción DIPECHO, que es el programa de preparación para desastres de la Comisión para la Ayuda Humanitaria de la Unión Europea (ECHO), que proporciona ayuda humanitaria directa a las poblaciones desamparadas, independientemente de su nacionalidad, religión, sexo u origen étnico. Colaborando con sus socios en el terreno, la Comisión actúa con rapidez para suministrar ayuda cuando se produce una catástrofe; y sigue ayudando a las regiones afectadas, incluso después de que han dejado de ser noticia y el interés mediático se ha desplazado a otra parte. Desde 1992, la Comisión ha financiado y coordinado operaciones humanitarias en más de 100 países fuera de la Unión Europea (Kawas et al. 2,010). La experiencia de los autores en la 
colaboración a diferentes instituciones nacionales e internacionales en esta materia fue de vital importancia en la elaboración de este trabajo. La realización de entrevistas a autoridades o técnicos de las principales organizaciones que forman parte del Sistema Nacional de Gestión de Riesgos y los sectores respectivos (educación, salud, medio ambiente, ordenamiento del territorio, etc.) fue con el objetivo de obtener información sobre sus líneas de acción y prioridades esenciales en la actualidad.

Las entrevistas se realizaron orientándose al campo específico de los actores y considerando las amenazas, vulnerabilidades, zonas prioritarias de intervención, estrategia y programas de la institución a cerca de preparativos para desastres, áreas temáticas específicas, niveles de intervención y relación con otros actores.

\section{RESULTADOS}

La Gestión del Riesgo a desastres en el país se inició a causa de los daños ocasionados por Huracán Mitch, que despertó el interés de las autoridades superiores, así como a los tomadores de decisión política y de la sociedad civil en el tema.

Aunque anteriormente el país había tenido situaciones similares como el Fifí 1974, Gilberto 1989 y otros, el nivel político y privado no se había apropiado de la variabilidad climática, en especial en el tema de frecuencia, duración e intensidad de estos fenómenos a su paso por la región.

Actualmente existe el SINAGER como sistema integrado de instituciones gubernamentales, civiles y privadas cuyos miembros tienen como responsabilidad primaria la gestión a nivel nacional de la infraestructura, energía, agricultura, academia, salud, recursos naturales, industria, economía, finanzas, planificación, logística, seguridad y telecomunicaciones. La empresa privada, sociedad civil y otras instituciones, todas cumplen con su responsabilidad en el tema y sector que les corresponde como responsables en el quehacer fundamental del país.

\section{Sobre la evolución del marco jurídico para GRRD}

El marco jurídico relacionado con la gestión del riesgo de desastres fue creado según Decreto Ley No. 33 del 30 de marzo de 1973, con el que se crea el Consejo Permanente de Emergencia Nacional (COPEN); luego, a través del Decreto No. 9$90 \mathrm{E}$ se crea el Reglamento de la Ley de Contingencias Nacionales, en el que se establece la Comisión Permanente de Contingencias (COPECO). 
El marco jurídico referencial tiene su fundamento, en forma generalizada, en la declaración constitucional que establece que la persona es el fin supremo de la Sociedad y del Estado y que todos tienen la obligación de respetarla y protegerla y, en función de este fin supremo, se garantiza la seguridad individual, la propiedad privada y las justas exigencias del bienestar general.

En forma específica, el marco jurídico se fundamenta en el Decreto No. 9-90E de fecha 12 de diciembre de 1990 (Ley de Contingencias Nacionales), el cual, en su Artículo 3, crea la Comisión Permanente de Contingencias COPECO, estableciéndole, en otra disposición y como objetivo fundamental, la adopción de políticas y medidas orientadas a atender a la población, rehabilitación y reconstrucción de las áreas dañadas por la incidencia de fenómenos naturales que afectan la actividad económica y el bienestar de la población, así como programar y desarrollar diferentes actividades, a fin de prevenir consecuencias negativas en las zonas de más incidencia de tales fenómenos.

De manera complementaria, el decreto en referencia fue reglamentado mediante Acuerdo No. 600-91 de fecha 26 de julio de 1991 y, mediante sus diferentes disposiciones, se establece, en términos puntuales, que la Comisión Permanente de Contingencias se constituye como un organismo responsable de coordinar los esfuerzos de los sectores públicos y privados para planificar, organizar, dirigir, ejecutar y controlar las acciones orientadas tanto a prevenir como a brindar ayuda a los sectores de población amenazados o que sean víctimas de problemas provocados por la alteración de fenómenos naturales en el país, los que de acuerdo con su magnitud sean calificados como emergencias, desastres o calamidades.

Tanto en la Ley como en el Reglamento, se prevé la participación de representantes de diversas instituciones y organismos públicos y privados que, en colaboración y bajo la coordinación de COPECO, se vinculan activamente en el momento de la atención de las emergencias, desarrollando sus funciones en los aspectos competentes a la organización que representan. Igualmente, las organizaciones que forman parte del Sistema tienen en sus diferentes instrumentos jurídicos, disposiciones específicas relativas a sus participaciones en la respuesta a emergencias y desastres.

En los últimos años se ha venido desarrollado un proceso de consulta y socialización para determinar un marco legal en la gestión del riesgo que permita evolucionar hacia una autoridad que pretende establecer el Sistema Nacional de Gestión de Riesgos (SINAGER), documento que fue completado y entregado en forma oficial al Congreso Nacional, el cual después de una amplia discusión fue aprobado y 
publicado en el diario oficial La Gaceta bajo Decreto 151-2009 el 26 de diciembre de 2009, Gaceta Numero 32098, convirtiendo a COPECO en el Organismo Coordinador del Sistema y al Comisionado Nacional como Secretario Ejecutivo. El SINAGER tiene por objeto establecer los principios, normativas orgánicas y funcionales que deberán regir en el Sistema, construyendo un marco legal orientado a prevenir y disminuir los riesgos de potenciales desastres provocados por fenómenos naturales o por las actividades humanas mediante gestiones de carácter preventivo y de recuperación. EI SINAGER se regula dentro del marco interinstitucional del Estado y comprende a todos los sectores de la sociedad hondureña.

En dicho marco se definen conceptos y se regulan acciones relacionadas con la prevención, mitigación, adaptación al cambio climático, manejo financiero del riesgo de desastres, preparación permanente y efectiva, la asistencia y ayuda humanitaria en caso de desastre y emergencia, y la rehabilitación y reconstrucción de las zonas afectadas, todo lo cual se encuentra comprendido dentro del concepto de "Gestión de Riesgos" a que se refiere esta Ley (Ley del SINAGER, Artículo 2). Actualmente se está trabajando en el reglamento de la Ley del SINAGER y se está planificando un proyecto para la socialización adecuada de la Ley y su Reglamento.

\section{Sobre las instituciones que trabajan en GRRD en el país}

En Honduras existen muchas instituciones que trabajan en este tema. Comenzando en el sector público, la COPECO, que funciona como el ente Coordinador del sector y de todas las demás instituciones miembros del Sistema de Gestión del Riesgo que apoyan en aspectos de prevención, atención, mitigación y rehabilitación ante un desastre.

Asimismo, está el sector académico, en especial el Instituto Hondureño de Ciencias de la Tierra de la Universidad Nacional Autónoma de Honduras que, aunque muy joven, ya ha logrado desarrollar varios estudios técnicos y de investigación en este tema que han contribuido al mejor conocimiento y caracterización de las zonas de mayor exposición a las amenazas en el país.

También la Sociedad Civil, que ha estado muy activa en los últimos 13 años a partir del Mitch, y con el apoyo de la cooperación internacional y países amigos, ejecutando actividades para la reducción del riesgo a desastres (RRD). Y, finalmente, el sector privado, que en muchos casos ha contribuido en estas actividades. 


\section{Sobre las debilidades en la GRRD en el país}

Las autoridades del país han estado más ocupadas en las áreas de atención y mitigación que en la prevención. Esta última ha sido más débil, debido al escaso interés por parte de las autoridades superiores del sector público, dado que a la fecha, los tomadores de decisiones, al parecer no son conscientes de la necesidad urgente de invertir en la prevención, a fin de lograr reducir las pérdidas de vidas humanas y de recursos físicos y socioeconómicos que hacen que el endeudamiento del gobierno central sea mayor cada vez que se presenta un fenómeno hidrometeorológico o de otra índole.

Otra de las debilidades encontradas durante la investigación es la falta de información básica estandarizada y con valores continuos y periódicos que permitan generar estudios e investigaciones profundas de la situación en una determinada región, poblado o departamento del país.

Muy pocas instituciones han desarrollado estudios a nivel de investigación; aun así, no existe una institución responsable de la producción de la información básica en todas las áreas de las ciencias relacionadas al tema. Las únicas que existen desarrollan y generan información de acuerdo a sus necesidades y al tema de su responsabilidad.

\section{Sobre las investigaciones en GRRD}

En Honduras se han desarrollado muchos estudios técnicos y de investigación, pero en muy pocos casos han sido tomados en cuenta para la gestión y preparación de planes a corto, mediano y largo plazo.

Se pueden mencionar los estudios de línea base en meteorología, hidrología, geofísica y de cambio climático, todos ellos actualizados, basados en información recién recopilada, gracias a algunas instituciones públicas que han tenido que generar la información durante muchos años de datos continuos de calidad que permiten el desarrollo de estudios de investigación en estos temas. Estos registros han servido en parte, pero falta mucho por producir, mayormente, investigación aplicada. En la Ley del SINAGER se impulsan mecanismos para la realización de investigación que sirven para la toma de decisiones en el campo de la GRRD.

\section{Sobre el aspecto financiero de los desastres}

Uno de los trabajos de análisis realizados identifica que las caídas del Producto Interno Bruto (PIB) del país desde el 1950 al 2010 se presentan especialmente dos 
meses después de la presencia de un fenómeno meteorológico (sea este El Niño, una depresión, tormenta o huracán) que ha producido efectos negativos durante su paso por Honduras. El gráfico No.1, que a continuación se detalla, muestra lo aseverado. (Kawas, 2007).

Gráfico No. 1: Ciclos en el PIB, constante entre 1950 y 2001

\section{Gráfico de los Ciclos del PIB constante 1950 y el 2001}

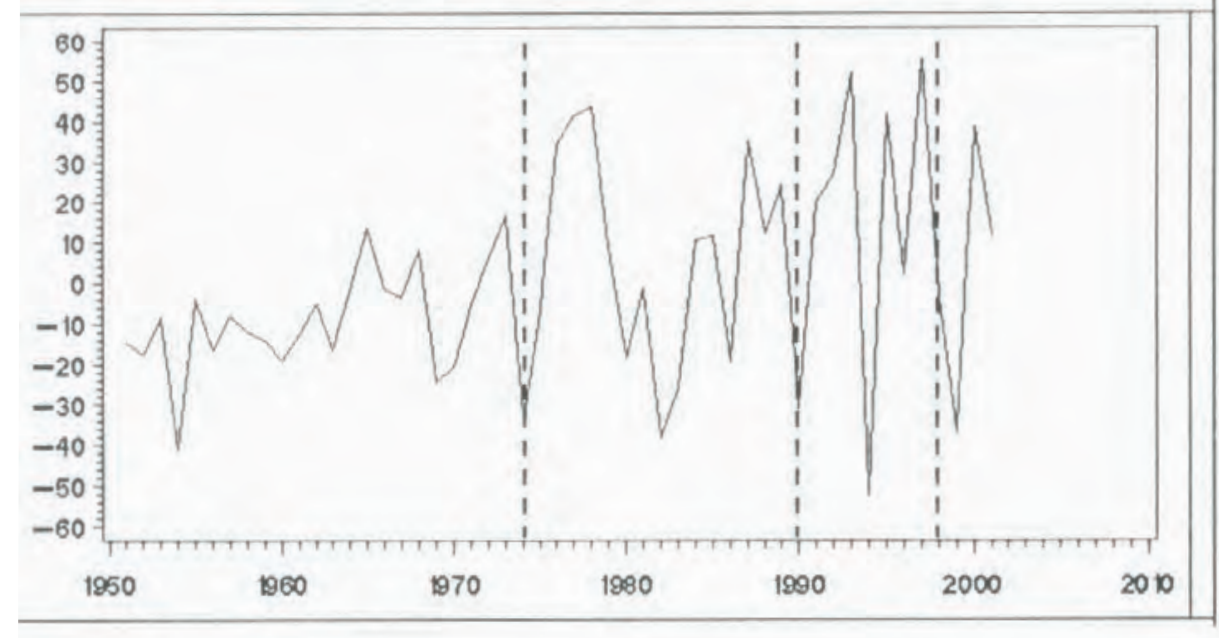

En el grafico 1 se puede ver que los principales picos de descenso del PIB han sido en 1954 (año en que sucedió lo que se conoce como "La Llena del 54", que produjo inundaciones que afectaron a gran parte del país), 1974 (el huracán Fifí), 1989 (el huracán Gilberto), 1994 (el huracán Gordon) y en 1998 (el huracán Mitch). Los demás descensos pertenecen al fenómeno de El Niño, que produce sequía en el Pacífico de todo Centroamérica. Ejemplo: 1982-1983, 1997-1998, etc.

Sobre las amenazas que han causado mayores daños y pérdidas en el país Las mayores amenazas a las que está expuesto el país son las de origen hidrometeorológico, entre ellas se pueden mencionar las naturales como los ciclones (depresiones, tormentas tropicales, huracanes), El Niño, las amenazas de la geodinámica terrestre como los terremotos, tsunamis y movimientos de ladera. Y las mareas y marejadas que también han afectado al país.

En el gráfico No. 2 se puede apreciar que las amenazas de origen hidrometeorológico, como los ciclones, son las que más han afectado al país en cuanto al número de muertos y damnificados. 
Gráfico No. 2: Muertos y damnificados por tipo de evento

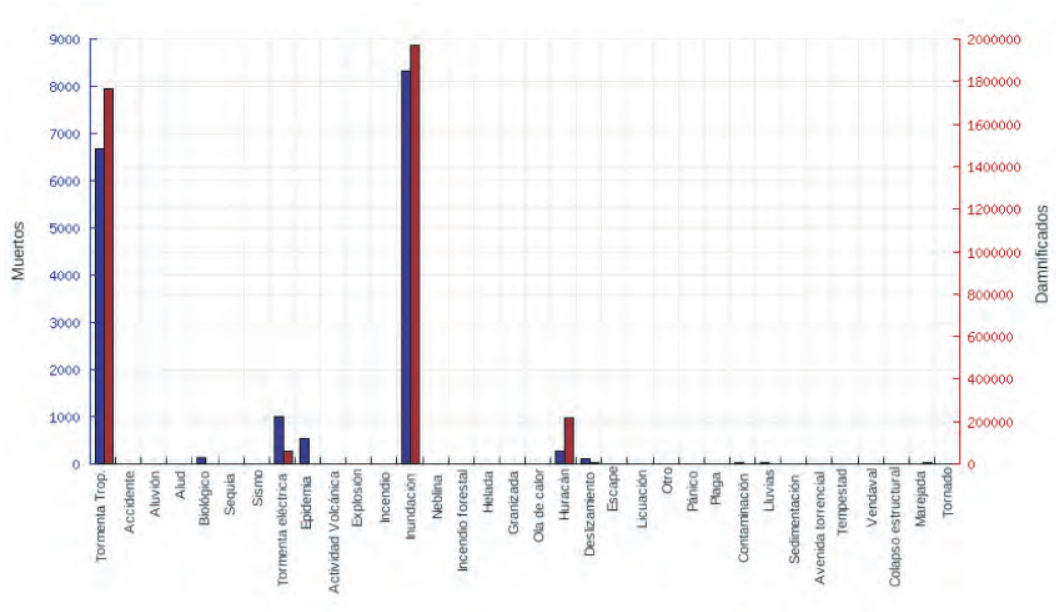

Muertos
Damnificados

Tipo de eventos

Fuente: elaborado a partir de la base de datos DesInventar de Honduras.

\section{Sobre la amenaza global del cambio climático en el país}

El cambio climático es un tema muy reciente a considerar en el país en cuanto a su estudio y análisis: está basado en la vulnerabilidad y ha permitido conocer, en alguna medida, de acuerdo a los estudios realizados, cómo estos cambios generan en muy pocos casos beneficios al país (aumento en la productividad agrícola en la zona norte), pero en su mayoría negativos en las regiones del centro, sur y pacifico, creando problemas energéticos, generando mayor contaminación y cambios en la flora y fauna alejando muchas especies conocidas y atrayendo a otras no muy comunes, en especial en las zonas costeras del Pacífico en la región centroamericana y el Caribe.

El futuro del país y de la región centroamericana, de acuerdo a los últimos escenarios generados para Honduras, es que se tendrá un ascenso de la temperatura en más de $1.5^{\circ} \mathrm{C}$, un aumento del nivel del mar en las costas del Océano Atlántico y Mar Caribe y mareas y marejadas frecuentes en el Pacífico. El índice de aridez irá incrementándose paulatinamente y extendiéndose a la mayor parte de nuestro territorio en los próximos 50 años, aumentando nuestra vulnerabilidad ante los efectos de estos cambios y sus consecuencias. La Secretaría de Recursos Naturales y Ambiente (SERNA), por medio de la Dirección Nacional de Cambio Climático, desarrolla acciones políticas y de planificación en la adaptación y mitigación de estos eventos. 


\section{Sobre la GRRD y la educación}

La educación y el conocimiento en GRRD son componentes que ayudan a elevar la capacidad de respuesta de una sociedad ante un desastre. En materia de educación se pueden señalar varios aspectos. Comenzando a nivel escolar y de media, las autoridades del Ministerio de Educación han aprobado acuerdos gubernamentales que obligan la inserción del tema de Gestión del Riesgo en toda actividad curricular del sistema educativo, lo cual permite a la población más joven entender y conocer de las amenazas, vulnerabilidades y riesgos a los que está expuesta su zona y el país en general por los diferentes fenómenos que se presentan.

Asimismo, en la educación superior, la Universidad Nacional Autónoma de Honduras, a través de su Instituto Hondureño de Ciencias de la Tierra, aprobó el desarrollo de un programa a nivel de maestría en gestión del riesgo y manejo de desastres que ha venido preparando un buen número de profesionales especialistas en el tema, que actualmente están trabajando en diferentes instituciones del estado, con la cooperación internacional y la sociedad civil, que atienden este tipo de emergencias.

\section{Sobre las zonas más vulnerables ante la ocurrencia de frecuentes amenazas}

Por su situación geográfica, en la región del Caribe Honduras está expuesto totalmente a los fenómenos naturales que se presentan, como ser ciclones y sismos, en especial en la zona norte y nororiental, mientras que en el centro y sur la exposición es a la sequía y al aumento del índice de aridez; y en la mayor parte del territorio nacional, por ser muy montañoso, la exposición es a los movimientos de ladera, producto del mal manejo de las cuencas hidrográficas, así como a la intervención del ser humano en las actividades agrícolas no tecnificadas que, acompañadas del mal uso del suelo y de las intensas lluvias durante la época lluviosa, son detonantes de los movimientos de laderas.

\section{Sobre la preparación en el país para hacerle frente a un desastre de grandes proporciones}

Definitivamente el país no está preparado para hacerle frente a un desastre de gran magnitud, como ser otro huracán clase IV, o un sismo de magnitud mayor de 5 en la escala Richter. Aunque se han desarrollado muchas acciones en la mayoría de la población y se han gastado enormes cantidades de dinero en la atención y rehabilitación y, agregado a ello, se ha mejorado el conocimiento del territorio 
nacional en este tema, la población en general está creciendo a un ritmo muy alto respecto a los estándares mundiales: la densidad de la población, en especial en ciertas aéreas del territorio, ha aumentado, reduciendo la capacidad instalada para la atención y recuperación ante un evento de esta naturaleza.

Acompañado a ello se siguen utilizando prácticas negativas y modos de vida contrarias a las regulaciones y capacidades de prevención, como la acumulación de basura en los lugares de mayor población a orillas de los ríos, degradación de cuencas, azolvamiento de los ríos y otras prácticas mal entendidas, así como la falta de recursos de diferentes tipos que hacen que el país siga siendo muy vulnerable a cualquier desastre de mediana y gran magnitud.

\section{DISCUSIÓN DE RESULTADOS}

La ruta que ha tenido la GRRD en el país desde hace 38 años con el inicio de un marco legal que ha venido evolucionando muy lentamente y con énfasis reactivo no proactivo, ha sido claramente tortuosa; sin embargo, el impacto del huracán Mitch (en el año 1998) provocó que los entes gubernamentales (especialmente la COPECO), con la ayuda de organismos de Cooperación Internacional iniciaran esfuerzos de GRRD; fue así como en el año 2000 se inició la ejecución de varios proyectos a través de organismos de cooperación y de países amigos, por ejemplo el PNUD, que fue de gran ayuda en la preparación de planes de emergencia de comunidades y municipios. En el año de 2001 se empieza a planificar el Proyecto de Mitigación de Desastres Naturales (PMDN), auspiciado por el Banco Mundial, que en un inicio fue dirigido por el Ministerio de Gobernación y Justicia y que sirvió de arranque para la realización de los primeros estudios de amenaza y vulnerabilidad, aunque no con el nivel técnico-científico que se requería.

Después del paso del huracán Mitch, muchas organizaciones han venido trabajando en diferentes acciones de GRRD, sin embargo, en la mayoría de los casos, los esfuerzos han sido mal enfocados, pues no han sido unificados ni estandarizados, tampoco han tenido la sistematización debida, lo que ha provocado redundancia en muchas de las acciones.

El impacto de eventos como tormentas tropicales y huracanes en el país es frecuente, pese a ello, la información en cuanto al impacto y valorización de pérdidas es muy escasa, o nula. La cantidad de damnificados por esos eventos en el periodo 1968-2010 asciende arriba de los cuatro millones de personas, la cual se puede considerar una cifra corta: de allí que los registros de los impactos sean necesarios para diferentes análisis (Kawas y Elvir, 2010). Esto demuestra la necesidad de 
mejorar en las capacidades de respuesta a desastres en el país y de ser conscientes de las amenazas y sus impactos. Uno de los sectores más afectados por estos eventos es el sector social y vivienda, con una gran cantidad de afectados, evacuados, reubicados y viviendas destruidas, lo que indica la alta vulnerabilidad de este sector.

Los estudios de amenazas, vulnerabilidad y riesgos en Honduras, en forma científica, prácticamente no existían antes de que el territorio fuese impactado por el huracán Mitch. A partir de esa fecha se pueden encontrar mapas de vulnerabilidad, de amenazas y de riegos. La participación de agencias gubernamentales, de países amigos y ONG's ha hecho posible la elaboración de varios estudios que incluyen mapas de riesgo, utilizando diferentes metodologías. Pese a los esfuerzos que se han hecho para elaborar algunos estudios técnico-científicos, falta mucho por hacer en el campo de la investigación, ya que no es fácil encontrar información de primera mano para hacer análisis de riegos, pues la información se encuentra diseminada en diferentes instituciones, no uniformada, ni estandarizada, lo cual hace muchas veces difícil su acceso.

El Índice de Riesgo Climático Global para Honduras, que es un análisis basado en datos confiables y disponibles de los impactos de los eventos meteorológicos extremos en las personas y su relación con los datos socioeconómicos, tiene un valor de 12.0 en el período de 1990 al 2008 y coloca al país en el tercer sitio mundial de riesgo climático en ese periodo (Argeñal, 2010). Esto pone de manifiesto que el país es altamente vulnerable al cambio climático, el cual es una amenaza global. Recientemente se ha aprobado la Estrategia Nacional de Adaptación al Cambio Climático y se ha empezado a trabajar en algunas acciones, sin embargo, el proceso ha sido bastante lento. Es una necesidad que en el país se vayan implementando algunas medidas de adaptación a nivel municipal, ya que en el mediano plazo los efectos del cambio climático se harán más notorios en algunas partes del país, sobre todo, en lo referente a disponibilidad de agua y a eventos hidrometeorológicos extremos.

En materia de educación, las acciones que se están realizando han sido aceptables, ya que éstas se han originado en todos los niveles del sector público; no obstante, en el sector privado, la temática, o no ha sido abordada, o se ha abordado superficialmente. Pese a las acciones realizadas, falta integrar en toda la currícula educativa el tema de gestión de riesgo.

Los diferentes estudios indican que desde las lecciones aprendidas con el huracán Mitch (hace 13 años), el país aun no tiene la capacidad de respuesta necesaria para 
poderle hacer frente a desastres de mediana y gran escala, por lo que es necesario reorientar la ruta de la GRRD y enfocar y acelerar el paso de las acciones en todos sus componentes.

\section{REFERENCIAS BIBLIOGRÁFICAS}

Agencia Suiza para el Desarrollo y la Cooperación (COSUDE). Proyecto Apoyo Local para el Análisis y Manejo de los Riesgos Naturales ALARN. Instrumentos de apoyo para el análisis y la gestión de riesgos naturales en el ámbito municipal de Nicaragua. 2002.

Argeñal, F. Inventario de la información disponible sobre amenazas, vulnerabilidad y riesgo climático en Honduras. 2010.

DesInventar. 2011. Sistema de inventario de efectos de desastres. http://online.desinventar.org/?lang=spa.

Kawas, N. Situación de los Recursos Hídricos de Honduras.2005 y 2007.

Kawas, N. et al. Documento de País, Honduras, DIPECHO VI. 2010.

Kawas, N; Elvir, O. Impacto de ciclones en Honduras, un resumen del periodo 19682010. 2010.

Ley del Sistema Nacional de Gestión de Riesgos (SINAGER) y de la Comisión Permanente de Contingencias (COPECO), 2009.

Motiño; S, Salgado; E, Murillo; A, Elvir; O. Estimación de daños y pérdidas por desastres de origen natural en el año 2,010 en el municipio del Distrito Central y los departamentos de Choluteca y Valle, Honduras C.A. 2010. 